\title{
Physical properties and lattice dynamics of bixbyite-type $\mathrm{V}_{2} \mathrm{O}_{3}$
}

\author{
Dominik A. Weber ${ }^{\text {a) }}$ \\ Institut für Chemie, Tecnische Universität Berlin, 10623 Berlin, Germany; and Physikalisch-Chemisches Institut, \\ Justus-Liebig-Universität Gießen, 35392 Gießen, Germany \\ Christian Schwickert \\ Institut für Anorganische und Analytische Chemie, Universität Münster, 84149 Münster, Germany \\ Anatoliy Senyshyn \\ Heinz Maier-Leibnitz Zentrum, Technische Universität München, 85748 Garching, Germany \\ Martin Lerch \\ Institut für Chemie, Tecnische Universität Berlin, 10623 Berlin, Germany \\ Rainer Pöttgen \\ Institut für Anorganische und Analytische Chemie, Universität Münster, 84149 Münster, Germany
}

(Received 8 December 2016; accepted 30 March 2017)

\begin{abstract}
Some time ago, we reported the synthesis of bixbyite-type $\mathrm{V}_{2} \mathrm{O}_{3}$, a new metastable polymorph of vanadium sesquioxide. Since, a number of investigations followed, dealing with different aspects like electronic and magnetic properties of the material, the deviation from ideal stoichiometry or the preparation of nanocrystals as oxygen storage material. However, most of the physical properties were only evaluated on a theoretical basis. Here, we report the lattice dynamics and physical properties of bixbyite-type $\mathrm{V}_{2} \mathrm{O}_{3}$ bulk material, which we acquired from physical property measurements and neutron diffraction experiments over a wide temperature range. Besides attributing different possible orientations of the magnetic moments for V1 and V2 to the identified antiferromagnetic (AFM) ground state with a Néel temperature of 38.1(5) K, we use a first order Grüneisen approximation to determine lattice-dependent parameters for the relatively stiff cubic lattice, and, amongst others identify the Debye temperature to be as low as $350 \pm 65 \mathrm{~K}$.
\end{abstract}

\section{INTRODUCTION}

Because of their structural diversity, abundant physical and chemical properties, and numerous potential applications, vanadium-based materials hold an exceptional position amongst transition metal-based compounds. Besides their use in electronic and optical devices, ${ }^{1}$ chemical sensors, ${ }^{2}$ as catalysts, ${ }^{3,4}$ and as cathode materials for high energy density lithium ion batteries, ${ }^{5-7}$ even binary vanadium oxides, due to their unique behavior, have been subject to various investigations. In particular, a large number of experimental and theoretical studies have already been conducted on the thermodynamically stable vanadium sesquioxide, as it presents a model system for a Mott-Hubbard transition. ${ }^{8-10}$ At low temperatures, this polymorph of $\mathrm{V}_{2} \mathrm{O}_{3}$ is an antiferromagnetic (AFM) insulator, crystallizing in a monoclinic structure (M1). At around $170 \mathrm{~K}$ it transforms into a paramagnetic conductor, undergoing a structural phase transition to the well-known rhombohedral corundumtype structure with a notably large $c / a$ ratio of $2.823 .^{9-14}$

Contributing Editor: Michael E. McHenry

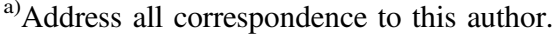

e-mail: dominik.weber@phys.chemie.uni-giessen.de

DOI: $10.1557 /$ jmr.2017.144
Regarding the inimitable characteristics of thermodynamically stable $\mathrm{V}_{2} \mathrm{O}_{3}$, the investigation of the physical properties of additional polymorphs of $\mathrm{V}_{2} \mathrm{O}_{3}$ is of fundamental interest. A few years ago, we synthesized a new metastable polymorph of vanadium sesquioxide that crystallizes in the bixbyite-type structure (space group $I a \overline{3}$ ). ${ }^{15}$ This polymorph of $\mathrm{V}_{2} \mathrm{O}_{3}$ has since been the subject of numerous investigations, including the synthesis of particles with urchin-like morphology by thermal decomposition of vanadyl ethylene glycolate ${ }^{16}$ or of phase pure nanocrystals by a colloidal route. ${ }^{17}$ Interestingly, these nanocrystals were only recently shown to reversibly incorporate up to two additional oxygen atoms per unit cell onto vacant anion sites in the crystal structure, ${ }^{18}$ fitting well with the predictions on intrinsic defects that we studied on density-functional theory (DFT) level. ${ }^{19}$ These vacant sites are located at the $16 c$ Wyckoff position in the bixbyite structure, which can be treated as an aniondeficient $2 \times 2 \times 2$ supercell of the fluorite structure with a quarter of the anions removed (Fig. 1). While the cations are distributed over two symmetry-inequivalent positions, $8 b$ and $24 d$, respectively, the anions populate the $48 e$ positions, giving a total of 80 atoms per unit cell $(Z=16)$.

Shortly after our first contribution on the new polymorph of $\mathrm{V}_{2} \mathrm{O}_{3}$, we theoretically studied the structural, 


\section{Fluorite}

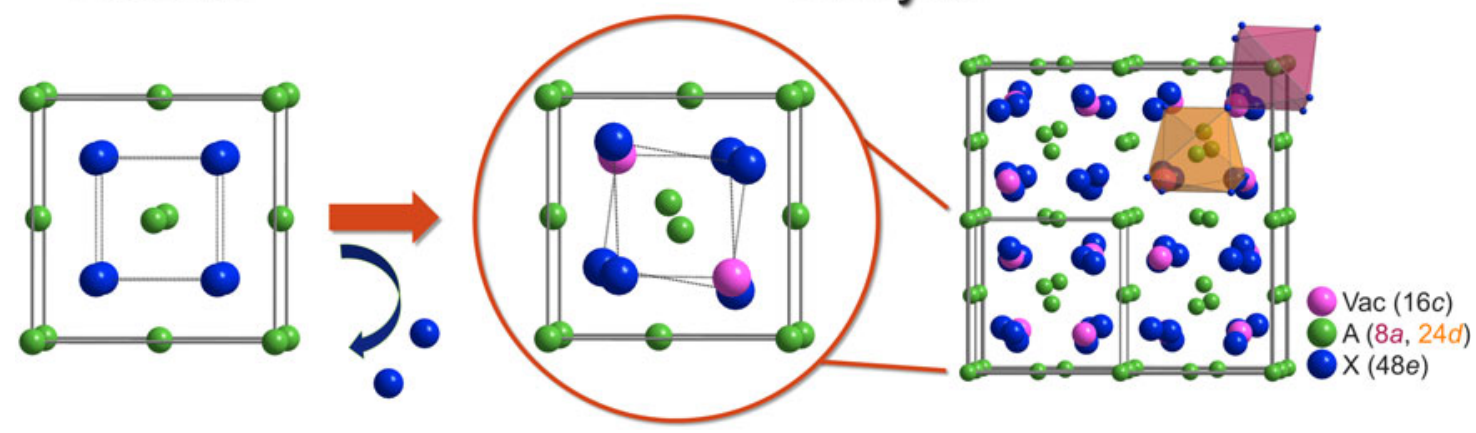

FIG. 1. Structural relationship between fluorite $f c c$ and bixbyite $b c c$ structure. Besides 16 ordered anion vacancies (16c position, pink), the bixbyite structure comprises two symmetry-inequivalent cation positions ( $8 a$, red and $24 d$, orange, respectively) as well as 48 equivalent anion positions (48e, blue). For $\mathrm{V}_{2} \mathrm{O}_{3}$ in this structure type, reversible incorporation of up to two additional oxygen atoms per unit cell is possible, utilizing the $16 c$ position.

thermodynamic, and electronic properties of the new phase amongst other possible polymorphs and found strong indications for the formation of a band gap in an AFM ground state. ${ }^{20}$

Up to that point, we were not able to isolate the phasepure material and physical property measurements were therefore carried out on samples that contained at least $18 \mathrm{wt} \%$ of the corundum-type polymorph as a side phase. Hence, we were not able to ascribe the observed phenomena for these samples solely to the bixbyitetype phase. Here, we present results of in-depth studies of the physical properties of phase-pure bixbyite-type $\mathrm{V}_{2} \mathrm{O}_{3}$ bulk material, which we recently were able to synthesize, ${ }^{21}$ and link them to results from temperaturedependent neutron and X-ray diffraction. For the first time, we determine the lattice dynamics of bixbyite-type $\mathrm{V}_{2} \mathrm{O}_{3}$, and confirm the AFM order of the material predicted by quantum-chemical calculations.

\section{EXPERIMENTAL SECTION}

\section{A. Sample preparation}

All reactions were carried out in a conventional tube furnace equipped with a corundum tube with an inner diameter of $50 \mathrm{~mm}$. The reaction gas was led directly onto the sample by a second corundum tube with an inner diameter of $5 \mathrm{~mm}$. The flow rate of the reaction gas [40 vol\% $\mathrm{H}_{2}$ in Ar (both Air Liquide, Dusseldorf, Germany), gas stream piped through a water-filled washing flask] was regulated with a parallel arrangement of two digital volumetric mass flow controllers (Brooks Instrument LLC, Hatfield, Pennsylvania) and set to $10 \mathrm{~L} / \mathrm{h}$. For each batch, $500 \mathrm{mg}$ of $\mathrm{V}_{2} \mathrm{~F}_{6} \cdot 4 \mathrm{H}_{2} \mathrm{O}$ or $\mathrm{V}_{2} \mathrm{~F}_{6} \cdot 6 \mathrm{H}_{2} \mathrm{O}$ (synthesized from vanadium powder and aqueous $\mathrm{H}_{2} \mathrm{SiF}_{6}^{22}$ ) were placed in a small alumina boat inside the tube and heated under flowing reaction gas for $2 \mathrm{~h}$ at $753 \mathrm{~K}$, applying a heating ramp of $300 \mathrm{~K} / \mathrm{h}$. After the dwell was complete, the furnace was swung open to allow for fast heat dissipation.
Simultaneously, the atmosphere was changed to $10 \mathrm{~L} / \mathrm{h}$ of dry argon (5.0, Air Liquide). After cooling, the grayishblack product was obtained without further treatment.

\section{B. Basic characterization}

The obtained products were characterized by X-ray powder diffraction (PANalytical X'Pert PRO MPD with $\mathrm{Cu} \mathrm{K}_{\alpha}$ radiation, PIXcel detector, PANalytical B.V., Almelo, The Netherlands) at ambient temperature. Structural refinements were performed in the same manner as described in the neutron diffraction section. Quantitative analysis of the oxygen content was performed by the well-established hot gas extraction method (LECO TC300/EF300, LECO Corp., St. Joseph, Michigan), using zirconia and corundum-type $\mathrm{V}_{2} \mathrm{O}_{3}$ as standards. The experimental error for this method amounts to $\approx 2 \%$ of the presented values (i.e., $\mathrm{V}_{2} \mathrm{O}_{3 \pm 0.06}$ ).

\section{Physical property measurements}

Magnetic measurements were performed in the temperature range of 2.5-305 K, using a Quantum Design Physical-Property-Measurement-System (Quantum Design, San Diego, California) with magnetic flux densities of up to $80 \mathrm{kOe}$. All measurements were carried out using the VSM option by packing the powdered sample $(15.642 \mathrm{mg})$ in a polypropylene capsule and attaching it to a brass sample holder. A heat capacity measurement was performed in the temperature range of 2.1-305 K, using the same Quantum Design Physical-Property-Measurement System. For the measurement, a compact sample was prepared and embedded into Apiezon $\mathrm{N}$ grease (Apiezon Products, M\&I Materials Ltd., Manchester, United Kingdom) to ensure thermal coupling with the sample holder platform.

\section{Neutron diffraction}

Elastic coherent neutron scattering experiments were performed at the Heinz Maier-Leibnitz Zentrum 
(Garching near Munich, Germany) on the high-resolution diffractometer SPODI. ${ }^{23}$ Monochromatic neutrons $(\lambda=$ $1.5482 \AA$ ) were obtained at a $155^{\circ}$ take-off angle, using the 551 reflection of a vertically-focused composite $\mathrm{Ge}$ monochromator. A vertical position-sensitive multidetector (300 mm effective height) consisting of $80{ }^{3} \mathrm{He}$ tubes and covering an angular range of $160 \mathrm{deg}$. $2 \theta$ was used for the data collection. Measurements were performed in Debye-Scherrer geometry. The powder sample (approximately $1 \mathrm{~cm}^{3}$ in volume) was filled into a thin-walled $(0.15 \mathrm{~mm})$ vanadium can of $10 \mathrm{~mm}$ in diameter, which was then mounted in the top-loading closed-cycle refrigerator. Helium 4.6 was used as a heat transmitter. The temperature was measured instantaneously, using two thin film resistance cryogenic temperature sensors $\left(\right.$ Cernox $^{\mathrm{TM}}$, Lake Shore Cyrotronics Inc., Westerville, $\mathrm{OH}$ ) and controlled by a temperature controller from LakeShore Cyrotronics Inc. Two-dimensional powder diffraction datasets were collected at fixed temperatures in the range of 4-320 K. Depending on the temperature, different exposure times were applied, i.e., to reveal the weak effect of magnetism in $\mathrm{V}_{2} \mathrm{O}_{3}$, the data at $4 \mathrm{~K}$ were collected for $21 \mathrm{~h}$, whilst shorter data collection times were applied at $60 \mathrm{~K}(10 \mathrm{~h})$ and $300 \mathrm{~K}(5 \mathrm{~h})$. Additionally, a number of diffraction patterns with short exposure times ( $30 \mathrm{~min}$ ) was collected at fixed temperatures in the temperature range 4 , $10,20,30, \ldots 320 \mathrm{~K}$. The collected 2D diffraction patterns were then corrected for geometrical aberrations and curvature of the Debye-Scherrer rings. ${ }^{24}$

Rietveld refinements were carried out using the software package FullProf Suite. ${ }^{25,26}$ The peak profile shape was modeled by a pseudo-Voigt function. The background of the diffraction pattern was fitted using a linear interpolation between selected data points in nonoverlapping regions. To deduce the evolution of the lattice parameters, the Rietveld method was applied for the analysis of the neutron data with no vanadium contribution, taking into account the anomalously low coherent neutron scattering length of the ${ }^{51} \mathrm{~V}$ isotope, which dominates in natural vanadium $(99.75 \%)$. For the coherent nuclear part of the scattering process, this low scattering length causes "blindness" of neutrons against vanadium. The magnetic form factor, on the other hand, depends on the magnetization distribution of the atoms, and therefore the detection of magnetic moments on natural vanadium atoms using nonpolarized neutron diffraction, is possible. The scale factor, the lattice parameter, the fractional coordinates of the atomic sites, and their isotropic displacement parameters, the zero angular shift, the profile shape parameters and the half width (Caglioti) parameters were varied during the fitting. Selected exemplary results for the data acquired at $T=4 \mathrm{~K}$ are presented in Table I together with the data from $\mathrm{X}$-ray diffraction at room temperature.
TABLE I. Experimental structural parameters of bixbyite-type $\mathrm{V}_{2} \mathrm{O}_{3}$ (obtained from neutron diffraction data at $T=4 \mathrm{~K}$ and X-ray diffraction data at $T=298 \mathrm{~K}$ ). The space group is $I a \overline{3}$ (No. 206). The structural data were modeled for the V1, V2, and $\mathrm{O} 1$ ions occupying the Wyckoff positions $8 a[0,0,0], 24 d[x, 0,1 / 4]$ and $48 e[x, y, z]$. Numbers in parentheses give estimated standard deviations in the last significant digit.

\begin{tabular}{|c|c|c|c|c|}
\hline \multirow{2}{*}{$\begin{array}{l}\text { Neutron diffraction @ } 4 \mathrm{~K} \\
\text { Atom site }\end{array}$} & \multicolumn{4}{|c|}{$a=9.3824(2) \AA, V=825.93(3) \AA^{3}$} \\
\hline & $x / a$ & $y / b$ & $z / c$ & $B_{\text {iso }}, \AA^{2}$ \\
\hline $\mathrm{V} 1$ & 0 & 0 & 0 & $0.6(4)$ \\
\hline $\mathrm{V} 2$ & $0.289(2)$ & 0 & $1 / 4$ & $0.6(4)$ \\
\hline $\mathrm{O} 1$ & $0.1394(1)$ & $0.1290(2)$ & $-0.0963(1)$ & $0.82(2)$ \\
\hline \multicolumn{5}{|c|}{$\mu=0.4(1) \mu_{\mathrm{B}} ; R_{\mathrm{p}}: 1.59 \%, R_{\mathrm{wp}}: 2.15 \%, R_{\mathrm{exp}}: 0.59 \%, \chi^{2}: 13.3$} \\
\hline \multicolumn{5}{|l|}{$\begin{array}{c}\mathrm{X}- \\
\text { ray diffraction @ } 298 \mathrm{~K}\end{array}$} \\
\hline Atom site & $x / a$ & $y / b$ & $z / c$ & $\begin{array}{c}B_{\text {isso, }} \\
\AA^{2}\end{array}$ \\
\hline V1 & 0 & 0 & 0 & $0.80(5)$ \\
\hline $\mathrm{V} 2$ & $0.28173(6)$ & 0 & $1 / 4$ & $0.95(4)$ \\
\hline $\mathrm{O} 1$ & $0.1426(2)$ & $0.1286(3)$ & $-0.0952(4)$ & $0.45(4)$ \\
\hline \multicolumn{5}{|c|}{$R_{\mathrm{p}}: 1.60 \%, R_{\mathrm{wp}}: 2.13 \%, R_{\mathrm{exp}}: 1.91 \%, \chi^{2}: 1.24$} \\
\hline
\end{tabular}

\section{RESULTS AND DISCUSSION}

\section{A. Syntheses and crystal chemistry}

Phase-pure bixbyite-type vanadium sesquioxide was successfully obtained as described in the experimental section. Quantitative oxygen analysis of the here-presented samples resulted in $31.8 \mathrm{wt} \%$ oxygen, leading to a composition of $\mathrm{V}_{2} \mathrm{O}_{2.98}$. This result is in fair agreement with the ideal bixbyite stoichiometry. The grayish-black product was structurally characterized using X-ray powder diffraction. The results of the Rietveld refinement are given in Fig. 2 and Table II.

The lattice parameter of $a=939.64(2) \mathrm{pm}$ shows no significant deviation $(\approx 0.02 \%)$ from the one we reported in our first contribution [939.47(2) pm]. ${ }^{15}$ Furthermore, the determined atomic parameters are in good agreement with the formerly reported ones.

\section{B. Magnetic properties}

The temperature dependent inverse susceptibility $\left[\chi^{-1}(T)\right.$ data] of $\mathrm{V}_{2} \mathrm{O}_{3}$, measured with magnetic flux densities of 1 and $10 \mathrm{kOe}$ (almost identical temperature dependence) is depicted in Fig. 3(a). The inset of Fig. 3(a) shows the low temperature regime of a temperature dependent susceptibility measurement in zero-field-cooled (ZFC)/field-cooled (FC) mode acquired with a low magnetic flux density of 100 Oe.

A least-squares fit of the inverse magnetic susceptibility data of the $10 \mathrm{kOe}$ measurement in the temperature range of $100-300 \mathrm{~K}$ with a modified Curie-Weiss law 


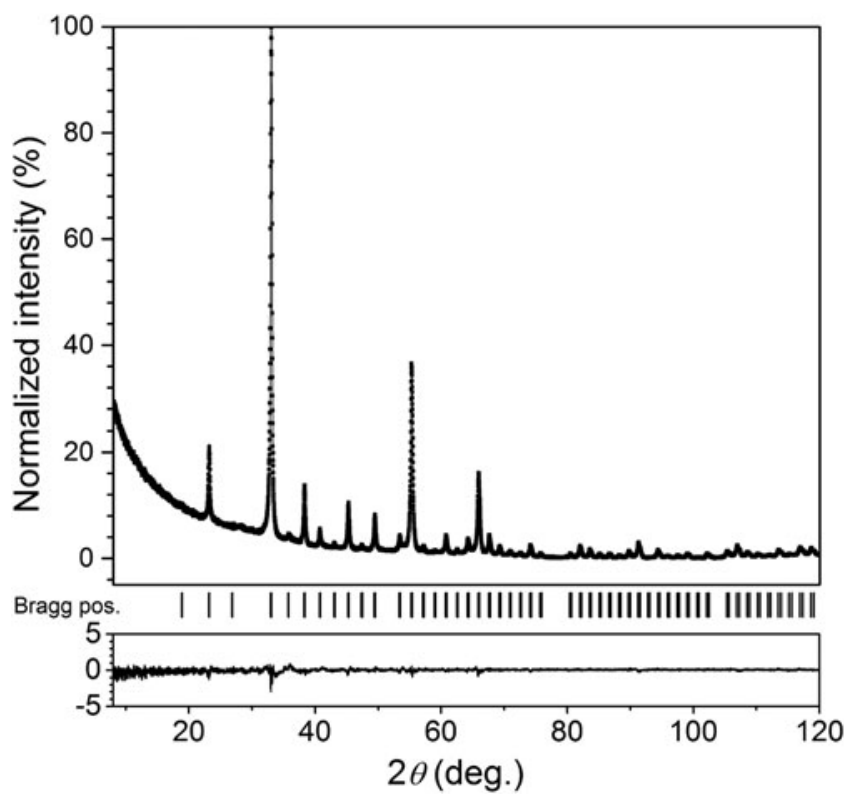

FIG. 2. X-ray powder diffraction diagram of phase-pure bixbyite-type $\mathrm{V}_{2} \mathrm{O}_{3}$ at room temperature with graphical results of the Rietveld refinement.

TABLE II. Numerical results of the Rietveld refinement against X-ray diffraction data at room temperature.

\begin{tabular}{lc}
\hline \hline Composition & $\mathrm{V}_{2} \mathrm{O}_{3}$ \\
\hline Structure type & Bixbyite \\
Space group & $I a \overline{3}$ \\
Lattice parameter & $a=939.64(2) \mathrm{pm}$ \\
Formula units & 16 \\
Unit cell volume (normalized to & $51.81 \times 10^{6} \mathrm{pm}^{3}$ \\
$\quad$ one formula unit) & $4.80 \mathrm{~g} / \mathrm{cm}^{3}$ \\
Calculated density & PANalytical X'Pert PRO MPD \\
Diffractometer & $\mathrm{Cu} \mathrm{K}_{\alpha}\left[\lambda_{1}=154.056 \mathrm{pm} ; \lambda_{2}=154.539\right.$ \\
Wavelength & $\left.\mathrm{pm} ;\left(\lambda_{2} / \lambda_{1}=0.5\right)\right]$ \\
& 8839 \\
Profile points & $5-120^{\circ}$ \\
$2 \theta$ range & 0.021 \\
$R_{\text {Bragg }}$ & 0.021 \\
$R_{\text {wp }}$ & 0.019 \\
$R_{\text {exp }}$ & 1.11 \\
$S$ (Goodness of fit) & \\
\hline \hline
\end{tabular}

$$
\chi^{-1}=\chi_{0}^{-1}+\frac{T-\theta}{C}
$$

yielded an experimental magnetic moment of $\mu_{\exp }=$ 1.72(1) $\mu_{\mathrm{B}}$ /vanadium-atom, a Weiss constant of $\theta_{\mathrm{P}}=-71.4(5) \mathrm{K}$ and a temperature independent contribution $\chi_{0}=6.49(1) \times 10^{-4} \mathrm{emu} / \mathrm{mol}$, where $\chi_{0}$ takes into account Van-Vleck and core corrections, as well as paramagnetic contributions of conduction band electrons. The experimental magnetic moment corresponds to the value for a free $\mathrm{V}^{4+}$ ion $\left(1.73 \mu_{\mathrm{B}}\right)$, assuming a spin-only high-spin state rather than a $\mathrm{V}^{3+}$ state. The Weiss
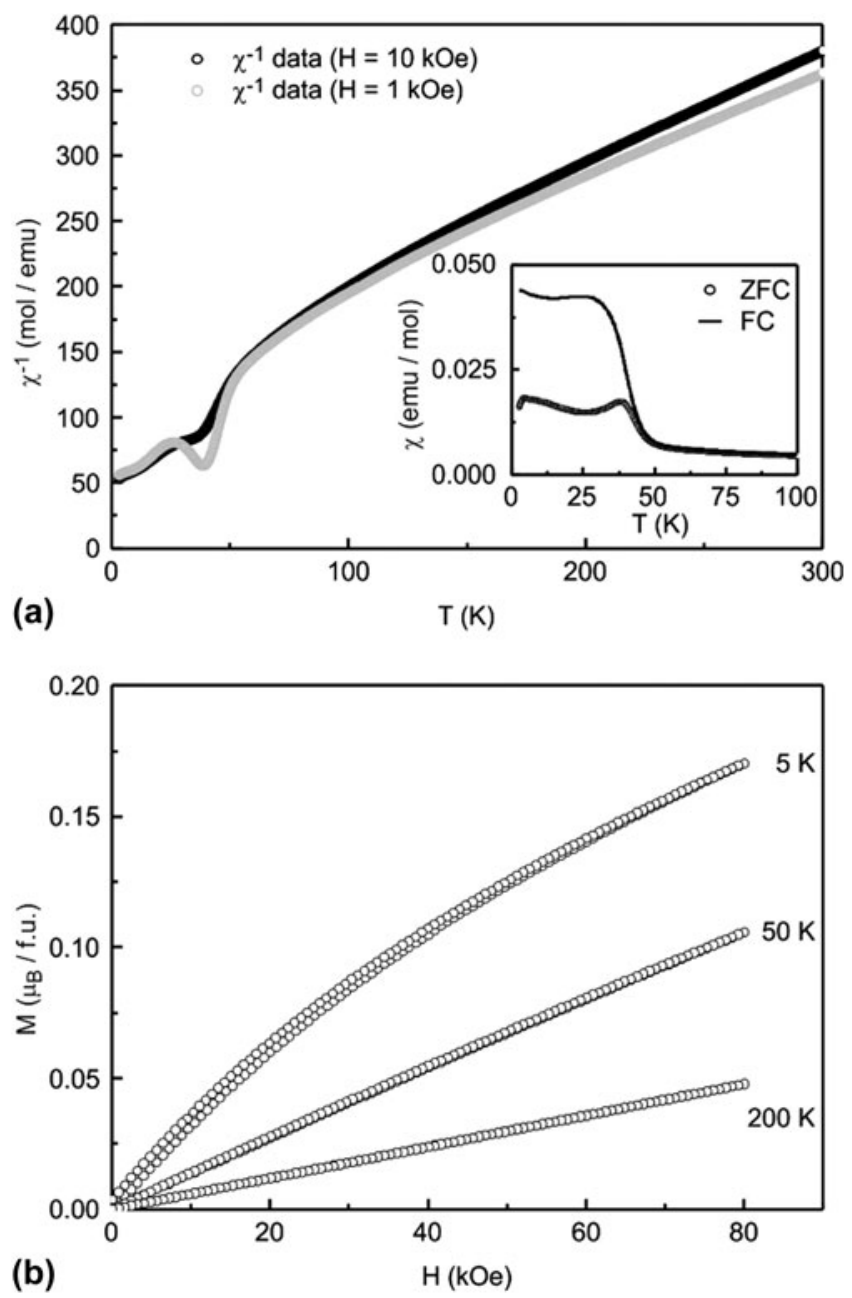

FIG. 3. (a) Temperature dependent inverse magnetic susceptibilities of cubic $\mathrm{V}_{2} \mathrm{O}_{3}$ measured at magnetic flux densities of 1 and $10 \mathrm{kOe}$. The inset depicts the low temperature regime of a temperature dependent magnetic susceptibility measurement of the $\mathrm{V}_{2} \mathrm{O}_{3}$ sample in ZFC/FC mode with a magnetic flux density of 100 Oe. (b) Magnetization isotherms of cubic $\mathrm{V}_{2} \mathrm{O}_{3}$ measured at 5, 50 and $200 \mathrm{~K}$ with magnetic flux densities up to $80 \mathrm{kOe}$.

constant is indicative of dominating AFM interactions in the paramagnetic domain. As will be discussed later, this behavior can be explained by frustration of the magnetic moments, leading to short-range order.

The Néel temperature $T_{\mathrm{N}}=38.1(5) \mathrm{K}$ was determined from the ZFC/FC measurement. As expected for paramagnetic materials, the magnetization isotherms [Fig. 3(b)] above the observed ordering temperature show a linear increase with the applied field. The $5 \mathrm{~K}$ isotherm exhibits no tendency of saturation, while showing a small hysteresis. From the course of the FC measurement along with the minute hysteresis of the $5 \mathrm{~K}$ magnetization isotherm [Fig. 3(b)], a canted, weakly ferromagnetic ground state has to be assumed.

The temperature dependence of the specific heat of the $\mathrm{V}_{2} \mathrm{O}_{3}$ sample is presented in Fig. 4. We observe no anomaly in the temperature window, where magnetic 


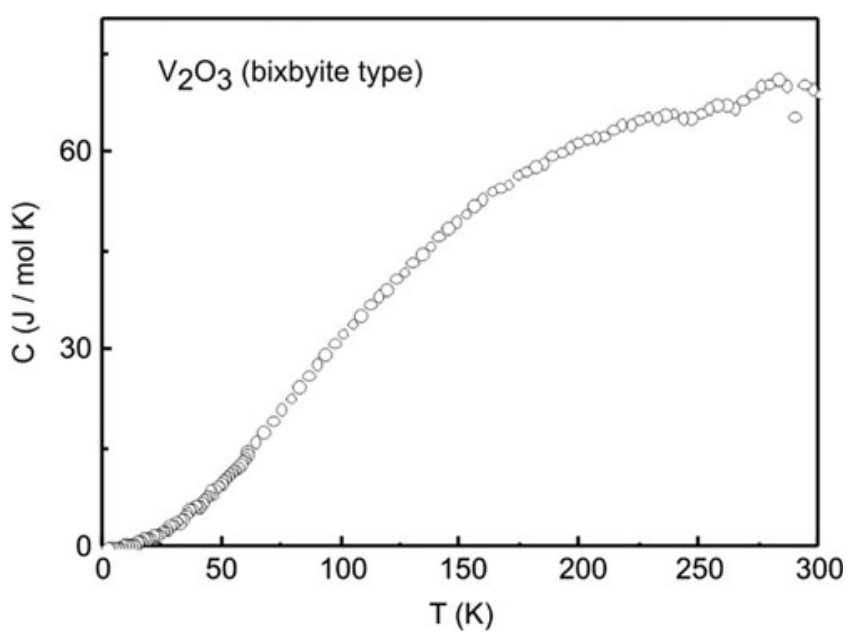

FIG. 4. Temperature dependence of the specific heat of cubic $\mathrm{V}_{2} \mathrm{O}_{3}$ measured in zero magnetic field.

ordering is evident in the susceptibility data. In view of the small value of the ordered moment, this is not surprising (see also the neutron diffraction data below). Thus, we only find a small value for the entropy change that is associated with the magnetic ordering process. This behavior is similar to that observed for $\mathrm{Sr}_{2} \mathrm{IrO}_{4}{ }^{27}$ Another important point concerns small degrees of vacancies or inhomogeneities within the oxygen substructure, which can also hamper long-range ordering due to both randomness and frustration of magnetic moments.

\section{Neutron diffraction studies}

The analysis of the neutron powder diffraction data at different temperatures revealed only reflections that are characteristic for a body centered cubic lattice (weak traces of aluminum from the walls of the closed-cycle refrigerator were present in the diffraction patterns at all temperatures), indicating the isostructurality of $\mathrm{V}_{2} \mathrm{O}_{3}$ within the whole studied temperature range. Due to the low coherent scattering length of vanadium in the natural isotope blend, its localization in the lattice on the basis of neutron data is quite challenging. An attempt to include vanadium into the refinement of the neutron powder diffraction data at room temperature resulted in a reduction of the $R$ factors by approximately $0.1-0.2 \%$. The accuracy of the vanadium localization from neutron and $\mathrm{X}$-ray diffraction data can be illustrated by the $x / a$ fractional coordinate for the V2 atom: the neutron diffraction experiment revealed $x / a=0.289(2)$, whereas X-ray powder diffraction enabled a determination of the V2 position at $x / a=0.28173(6)$ (see Table I), which demonstrates an about two orders of magnitude better resolution for the latter.

The temperature dependent evolution of the lattice parameter of cubic $\mathrm{V}_{2} \mathrm{O}_{3}$ was determined by Rietveld analysis of the "short" neutron powder diffraction data sets collected in the temperature range between 4 and $320 \mathrm{~K}$. The resulting thermal dependence of the lattice parameter was obtained by combining the obtained results with high-temperature lattice parameters, we obtained earlier. ${ }^{15}$ The results are shown in Fig. 5. Both, low- and high-temperature data sets were found to be in good agreement, thus indicating that the structure of cubic $\mathrm{V}_{2} \mathrm{O}_{3}$ is quite stiff to temperature changes, i.e., heating from 4 to $850 \mathrm{~K}$ resulted in a minor increase of the lattice parameter by approximately $0.04 \AA(\approx 0.4 \%)$.

The first order Grüneisen approximation was applied to interpret the obtained temperature dependence of the structure of cubic $\mathrm{V}_{2} \mathrm{O}_{3}$. It can be described as

$$
V(T)=V_{0}+\frac{\gamma}{K_{T}} U(T),
$$

where $V_{0}$ denotes the hypothetical cell volume at zero temperature, $\gamma$ is the Grüneisen constant, $K$ is the bulk modulus, and $U$ is the internal energy of the system. Both, the Grüneisen constant and the bulk modulus are supposed to be temperature-independent and the Debye approximation for the internal energy $U$ in Eq. (1) has the form

$$
U(T)=U_{\mathrm{D}}(T)=\left[9 N k_{\mathrm{B}} T\left(\frac{T}{\theta_{\mathrm{D}}}\right)^{3} \int_{0}^{\theta_{\mathrm{D}} / T} \frac{x^{3}}{\mathrm{e}^{x}-1} \mathrm{~d} x\right],
$$

with the characteristic temperature $\theta_{\mathrm{D}}$ (Debye temperature), the number of atoms in the unit cell $N$ and the Boltzmann constant $k_{\mathrm{B}}$, providing a reasonable description for the cell volume, similar to the procedure applied to data on $\mathrm{FeSi}^{28}$

Least-squares nonlinear fitting of the experimental data according to Eqs. (1) and (2) resulted in an adequate description of the lattice parameter of cubic $\mathrm{V}_{2} \mathrm{O}_{3}$ by the first order Grüneisen approximation with $V_{0}=825.8 \pm$ $0.1 \AA^{3}, \gamma / \mathrm{K}=4.4 \pm 0.1 \times 10^{-12} \mathrm{~Pa}^{-1}$ and $\theta_{\mathrm{D}}=350 \pm$ $65 \mathrm{~K}$ (see Fig. 5). The cubic modification of $\mathrm{V}_{2} \mathrm{O}_{3}$ is characterized by a relatively low Debye temperature; for the rhombohedral corundum-type modification of $\mathrm{V}_{2} \mathrm{O}_{3}$, a Debye temperature ranging from 575 to $643 \mathrm{~K}$ has been reported. ${ }^{29}$ On the other hand, the compound shows a weak thermal expansion: The thermal expansion coefficient calculated as $a_{l}(T)=\frac{\partial \ln l(T)}{\partial T}=\frac{1}{3} \frac{\partial \ln V(T)}{\partial T}$ yielded $a_{l} \sim 5.8 \mathrm{~K}^{-1}$ at the proximity of the phase transition to the corundum-type phase at approximately $823 \mathrm{~K}^{15}$

Besides the weak anharmonicity effects, cubic $\mathrm{V}_{2} \mathrm{O}_{3}$ is known to order magnetically. ${ }^{15}$ A previously performed evaluation of the lattice dimensions versus temperature revealed no sufficient magnetoelastic couplings to the lattice in $\mathrm{V}_{2} \mathrm{O}_{3}$. Additionally, the inspection of the "short" neutron powder diffraction data sets provided no 


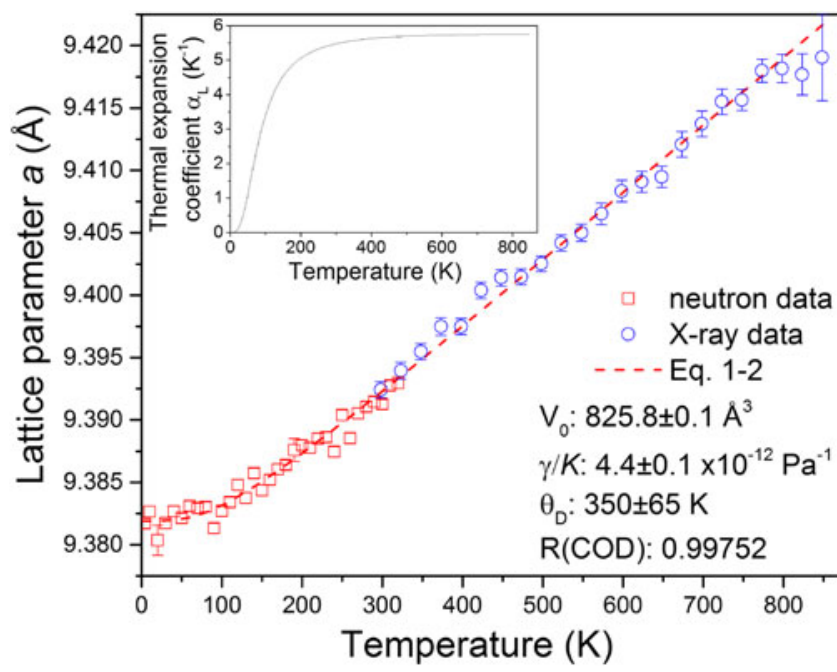

FIG. 5. Temperature evolution of the lattice parameter in cubic $\mathrm{V}_{2} \mathrm{O}_{3}$; neutron data are shown in red, X-ray data ${ }^{15}$ in blue color. The scattered lines correspond to the results of data fitting by Eqs. (1) and (2); the calculated thermal expansion coefficient is shown in the inset.

visible indication for a long range magnetic order below $T_{\mathrm{C}}$. Only a detailed evaluation of the "long" diffraction data sets collected at 4 and $60 \mathrm{~K}$ yielded weak additional neutron intensities on top of the nuclear reflections at low angles (see Fig. 6), which can be an evidence for long range magnetic ordering, with a magnetic lattice that has identical metrics as the nuclear one (propagation vector $\mathbf{k}=[0,0,0])$.

Bixbyite-type $\mathrm{V}_{2} \mathrm{O}_{3}$ comprises two crystallographically inequivalent vanadium positions. An analysis of the magnetic representations was performed by the Bertaut method ${ }^{30}$ implemented in the BasIreps software, ${ }^{31}$ revealing 8 irreducible representations that form a little group $\mathbf{G}_{\mathbf{k}}$ (coinciding with the $I a \overline{3}$ symmetry and leaving the propagation vector $\mathbf{k}$ invariant). Due to the weak thermal expansion and the weak magnitude of the observed magnetic effects, the evaluation of the magnetic structures was performed on the differential diffraction pattern in the $2 \theta$ angular range of $10-35^{\circ}$. Magnetic structure models based on 1D irreducible representations were constructed in different configurations, but their refinement did not yield an adequate description of the magnetic intensities. 3D irreducible representations, on the other hand, apply fewer constraints to the orientation and magnitude of the magnetic moments in bixbyite-type $\mathrm{V}_{2} \mathrm{O}_{3}$. However, no consistent conclusion about the magnetic structure can be made from the least-squares minimization due to convergence and correlation issues, which can be related to the restrictions applied to the highly symmetric lattice regarding determination of the magnetic moment orientations from powder diffraction data. ${ }^{32}$

To describe the obtained residual intensities, the iterative approach (assuming a 2D collinear AFM

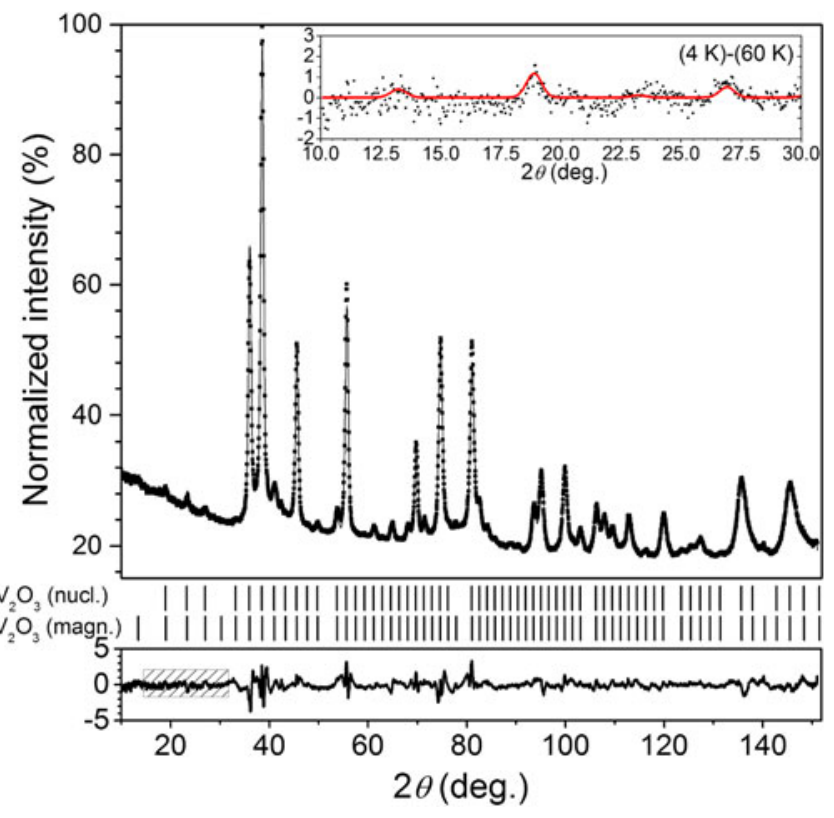

FIG. 6. Graphical results of the Rietveld refinement against neutron powder diffraction data of bixbyite-type $\mathrm{V}_{2} \mathrm{O}_{3}$ at $4 \mathrm{~K}$; calculated positions of Bragg reflections for the nuclear and the magnetic contribution are shown by the top and bottom row of vertical tick marks. The inset illustrates the low temperature section of the normalized differential diffraction patterns collected at 4 and $60 \mathrm{~K}$. The red line corresponds to the model for the magnetic contribution.

structure for $\mathrm{V}_{2} \mathrm{O}_{3}$ ) has been applied. The magnetic moments for the $\mathrm{V}^{3+}$ sites were aligned in 2 states: either alongside or opposite to the $c$ axis. With 16 formula units in bixbyite-type $\mathrm{V}_{2} \mathrm{O}_{3}$, the number of possible configurations is limited to $2 .{ }^{16} \mathrm{An}$ assumption about the AFM ground state at $4 \mathrm{~K}$ (supported by the magnetization measurements) further reduces the number of configurations to 12,870 . Each configuration with a predefined orientation of the magnetic moments was evaluated by Rietveld refinement, where the amplitude of the magnetic moments was constrained to be the same for both V1 and V2, and was allowed to vary.

Due to the low magnitude of the observed effects and, correspondingly, large fit residuals, the sum of the magnetic $R$ factor and $\chi^{2}$ were used as a figure of merit (the use of $R_{\mathrm{p}}$ and $R_{\mathrm{wp}}$ does not change the results). Four configurations (shown in Fig. 7) possessing equal values for $R_{\mathrm{m}}+\chi^{2}$ have been identified and their accuracy cannot further be judged on the basis of the present neutron powder diffraction data (similar models can also be drawn, assuming an FM direction opposite to the $c$ axis; indistinguishable by powder diffraction). The magnetic moments of the V1 sites have been found to be oriented ferromagnetically along the $c$ axis, whilst the moments of the V2 sites are located either alongside or opposite to the $c$ axis to maintain zero net magnetization. The amplitude of the magnetic moments was refined to 


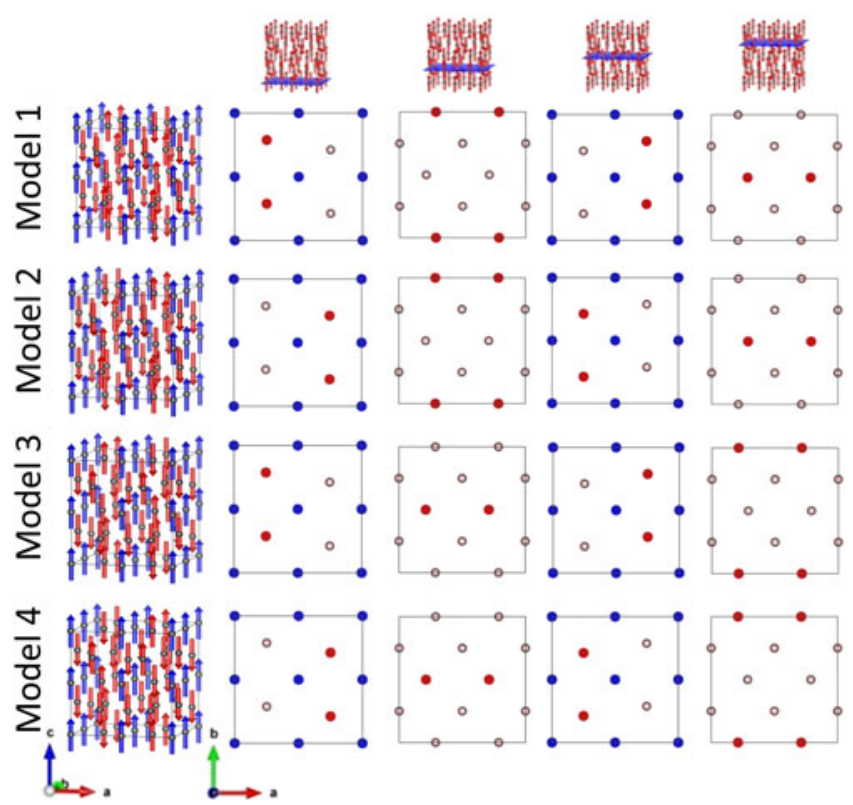

FIG. 7. Obtained variances of the collinear AFM ground state in bixbyite $\mathrm{V}_{2} \mathrm{O}_{3}$ based on Rietveld modeled data at $4 \mathrm{~K}$ (column 1). The magnetic moments on the V1 site are shown in blue color, magnetic moments on V2 are in red. A sketch of the magnetic moment orientation at four distinct 001 planes is shown in columns $2-5$, solid points depict moment orientation along the $c$ axis.

0.71(3) $\mu_{\mathrm{B}}$, using differential data and 0.4(1) $\mu_{\mathrm{B}}$, using a completed data set at $4 \mathrm{~K}$. Anyways, these values are considerably lower than the expected moment for $\mathrm{V}^{3+}$ (approximately $2.8 \mu_{\mathrm{B}}^{33}$ ). We already reported some discrepancies, earlier ${ }^{15}$ and associated them with deviations from the $\mathrm{V}^{3+}$ state of the vanadium ions, which we also rendered possible by theoretical calculations. ${ }^{19}$ On the other hand, magnetic interactions may undergo a geometrical frustration in bixbyite-type $\mathrm{V}_{2} \mathrm{O}_{3}$, with the $\mathrm{V} 1$ and V2 ions located on the vertices of trigonal and hexagonal frameworks. One also has to mention that a constraint of the magnetic moments on V1 and V2 leads to an AFM lattice with zero net magnetization, whereas a small discrepancy in the moment amplitudes between the V1 and V2 magnetic sites (initially permitted by symmetry) may result in the appearance of a weak ferromagnetic moment. This is in excellent agreement with the absence of a lambda type anomaly in the specific heat data (Fig. 4).

\section{CONCLUSIONS}

The magnetic properties of bixbyite-type $\mathrm{V}_{2} \mathrm{O}_{3}$ have been studied and revealed an experimental magnetic moment of $\mu_{\exp }=1.72(1) \mu_{\mathrm{B}} /$ vanadium-atom, which interestingly corresponds to a free $\mathrm{V}^{4+}$ ion $\left(1.73 \mu_{\mathrm{B}}\right)$, rather than $\mathrm{V}^{3+}$. The observed Weiss constant of $\theta_{\mathrm{P}}=-71.4(5) \mathrm{K}$ is indicative for AFM interactions in the paramagnetic domain and a canted, weakly AFM ground state seems to be present. A Néel temperature of $T_{\mathrm{N}}=38.1(5) \mathrm{K}$ was determined. Furthermore, only a very small value for the entropy change during magnetic ordering is present, most probably because of randomness and frustration of the magnetic moments, hampering long-range ordering in the material.

Using high-resolution neutron powder diffraction at low and intermediate temperatures, isostructurality of $\mathrm{V}_{2} \mathrm{O}_{3}$ has been confirmed in the whole temperature range from 4 to $320 \mathrm{~K}$. The cubic $\mathrm{V}_{2} \mathrm{O}_{3}$ is characterized by a rather low thermal expansion in the whole range of existence (below $823 \mathrm{~K}$ ), which can be fairly well described by a first order Grüneisen approximation. For the first time, the hypothetical zero Kelvin value for the cell volume $V_{0}$ as well as the temperature independent $\gamma / K$ ratio and Debye temperature $\theta_{\mathrm{D}}$ have been determined to be as large as $825.8 \pm 0.1 \AA^{3}, 4.4 \pm$ $0.1 \times 10^{-12} \mathrm{~Pa}^{-1}$, and $350 \pm 65 \mathrm{~K}$.

Based on the iterative approach, 12,870 different AFM configurations of the $\mathrm{V}^{3+}$ magnetic moment arrangements adopting zero net magnetization have been evaluated using Rietveld refinements. Based on the fit residuals, four possible configurations have been chosen, where all adopt FM arrangement of the V1 magnetic moments and ferrimagnetic order of V2 (with a weak FM component opposite to that of V1). A weak ferrimagnetic component in bixbyite-type $\mathrm{V}_{2} \mathrm{O}_{3}$ can be tuned by the discrepancy of the V1 and V2 magnetic moments and the weak ferromagnetism can be attributed to the different order temperatures (and, correspondingly, saturation behavior) for the V1 and V2 magnetic ions.

Due to the weak magnitude of the ordered moments, an accurate determination of their arrangement between different vanadium sites would require neutron diffraction experiments on single crystals.

\section{ACKNOWLEDGMENT}

Financial support from the German Research Foundation (DFG) within the priority program SPP 1415 (LE 781/11-2) is gratefully acknowledged. The authors further gratefully acknowledge the financial support provided by FRM II to perform the neutron scattering measurements at the Heinz Maier-Leibnitz Zentrum (MLZ), Garching, Germany.

\section{REFERENCES}

1. J. Muster, G.J. Kini, J.G. Park, and M. Burghard: Electrical transport through individual vanadium pentoxide nanowires. Adv. Mater. 12, 420 (2000).

2. P. Liu, S-H. Lee, H.M. Cheong, C.E. Tracy, J.R. Pitts, and R.D. Smith: Stable $\mathrm{Pd} / \mathrm{V}_{2} \mathrm{O}_{5}$ optical $\mathrm{H}_{2}$ sensor. J. Electrochem. Soc. 149, H76 (2002).

3. J. Haber: Fifty years of my romance with vanadium oxide catalysts. Catal. Today 142, 100 (2009). 
4. C. Hess: Nanostructured vanadium oxide model catalysts for selective oxidation reactions. ChemPhysChem 10, 319 (2009).

5. P.P. Prosini, Y. Xia, T. Fujieda, R. Vellone, M. Shikano, and T. Sakai: Performance and capacity fade of $\mathrm{V}_{2} \mathrm{O}_{5}$-lithium polymer batteries at a moderate-low temperature. Electrochim. Acta 46, 2623 (2001).

6. Y. Shi, Z.J. Zhang, D. Wexler, S. Chou, J. Gao, H.D. Abruna, H. Li, H. Liu, Y. Wu, and J. Wang: Facile synthesis of porous $\mathrm{V}_{2} \mathrm{O}_{3} / \mathrm{C}$ composites as lithium storage material with enhanced capacity and good rate capability. J. Power Sources 275, 392 (2015).

7. L. Jiang, Y. Qu, Z. Ren, P. Yu, D. Zhao, W. Zhou, L. Wang, and $\mathrm{H}$. Fu: In situ carbon-coated yolk-shell $\mathrm{V}_{2} \mathrm{O}_{3}$ microspheres for lithium-ion batteries. ACS Appl. Mater. Interfaces 7, 1595 (2015).

8. N.F. Mott: Metal-insulator Transition (Taylor and Francis Ltd, London, 1974).

9. K. Held, G. Keller, V. Eyert, D. Vollhardt, and V.I. Anisimov: Mott-Hubbard metal-insulator transition in paramagnetic $\mathrm{V}_{2} \mathrm{O}_{3}$ : An LDA + DMFT(QMC) study. Phys. Rev. Lett. 86, 5345 (2001).

10. P. Hansmann, A. Toschi, G. Sangiovanni, T. Saha-Dasgupta, S. Lupi, M. Marsi, and K. Held: Mott-Hubbard transition in $\mathrm{V}_{2} \mathrm{O}_{3}$ revisited. Phys. Status Solidi B 250, 1251 (2013).

11. L.W. Finger and R.M. Hazen: Crystal structure and isothermal compression of $\mathrm{Fe}_{2} \mathrm{O}_{3}, \mathrm{Cr}_{2} \mathrm{O}_{3}$, and $\mathrm{V}_{2} \mathrm{O}_{3}$ to 50 kbars. J. Appl. Phys. 51, 5362 (1980).

12. D.B. McWhan and J.B. Remeika: Metal-insulator transition in $\left(\mathrm{V}_{1-x} \mathrm{Cr}_{x}\right)_{2} \mathrm{O}_{3}$. Phys. Rev. B: Condens. Matter Mater. Phys. 2, 3734 (1970).

13. P.D. Dernier and M. Marezio: Crystal structure of the lowtemperature antiferromagnetic phase of $\mathrm{V}_{2} \mathrm{O}_{3}$. Phys. Rev. B: Condens. Matter Mater. Phys. 2, 3771 (1970).

14. R.M. Moon: Antiferromagnetism in $\mathrm{V}_{2} \mathrm{O}_{3}$. Phys. Rev. Lett. 25, 527 (1970).

15. D. Weber, A. Stork, S. Nakhal, C. Wessel, C. Reimann, W. Hermes, A. Müller, T. Ressler, R. Pöttgen, T. Bredow, R. Dronskowski, and M. Lerch: Bixbyite-type $\mathrm{V}_{2} \mathrm{O}_{3}$ : A metastable polymorph of vanadium sesquioxide. Inorg. Chem. 50, 6762 (2011).

16. A. Bergerud, R. Buonsanti, J.L. Jordan-Sweet, and D.J. Milliron: Synthesis and phase stability of metastable bixbyite $\mathrm{V}_{2} \mathrm{O}_{3}$ colloidal nanocrystals. Chem. Mater. 25, 3172 (2013).

17. Y. Xu, L. Zheng, C. Wu, F. Qi, and Y. Xie: New-phased metastable $\mathrm{V}_{2} \mathrm{O}_{3}$ porous urchinlike micronanostructures: Facile synthesis and application in aqueous lithium ion batteries. Chem. Eur. J. 17, 384 (2011).

18. A. Bergerud, S.M. Selbach, and D.J. Milliron: Oxygen incorporation and release in metastable bixbyite $\mathrm{V}_{2} \mathrm{O}_{3}$ nanocrystals. ACS Nano 10, 6147 (2016).
19. C. Reimann, D. Weber, M. Lerch, and T. Bredow: Nonstoichiometry in bixbyite-type vanadium sesquioxide. J. Phys. Chem. C 117, 20164 (2013).

20. C. Wessel, C. Reimann, A. Müller, D. Weber, M. Lerch, T. Ressler, T. Bredow, and R. Dronskowski: Electronic structure and thermodynamics of $\mathrm{V}_{2} \mathrm{O}_{3}$ polymorphs. J. Comput. Chem. 33, 2102 (2012).

21. T. Lüdtke, D. Weber, A. Schmidt, A. Müller, C. Reimann, N. Becker, T. Bredow, R. Dronskowski, T. Ressler, and M. Lerch: Synthesis and characterization of metastable transition metal oxides and oxide nitrides. Z. Kristallogr. 232(1-3), 3-14 (2017).

22. S. Nakhal, D. Weber, E. Irran, M. Lerch, B. Schwarz, and $\mathrm{H}$. Ehrenberg: Synthese und Kristallstrukturen der neuen Metallfluoridhydrate $\mathrm{V}_{2} \mathrm{~F}_{6} \cdot 4 \mathrm{H}_{2} \mathrm{O}$ und $\mathrm{Mn}_{3} \mathrm{~F}_{8} \cdot 2 \mathrm{H}_{2} \mathrm{O}$. Z. Anorg. Allg. Chem. 636, 2061 (2010).

23. M. Hölzel, A. Senyshyn, and O. Dolotko: SPODI: High resolution powder diffractometer. Journal of Large-Scale Research Facilities 1, A5 (2015).

24. M. Hölzel, A. Senyshyn, N. Jünke, H. Boysen, W. Schmahl, and H. Fuess: High-resolution neutron powder diffractometer SPODI at research reactor FRM II. Nucl. Instrum. Methods Phys. Res., Sect. A 667, 32 (2012).

25. J. Rodriguez-Carvajal: Recent advances in magnetic structure determination by neutron powder diffraction. Physica B 192, 55 (1993).

26. J. Rodriguez-Carvajal: Recent developments of the program FULLPROF. Commission on Powder Diffraction Newsletter 26, 12 (2001).

27. N.S. Kini, A.M. Strydom, H.S. Jeevan, C. Geibel, and S.J. Ramakrishnan: Transport and thermal properties of weakly ferromagnetic $\mathrm{Sr}_{2} \mathrm{IrO}_{4}$. J. Phys.: Condens. Matter 18, 8205 (2006).

28. L. Vočadlo, K.S. Knight, G.D. Price, and I.G. Wood: Thermal expansion and crystal structure of FeSi between 4 and $1173 \mathrm{~K}$ determined by time-of-flight neutron powder diffraction. Phys. Chem. Miner. 29, 132 (2002).

29. Semiconductors, 'Non-Tetrahedrally Bonded Binary Compounds II', Landolt-Börnstein, Group III Condensed Matter Subvolume D, Vol. 41, O. Madelung, U. Rössler, and M. Schulz, eds. (Springer, New York, USA, 1969).

30. E.F. Bertaut: Representation analysis of magnetic structures. Acta Crystallogr., Sect. A: Cryst. Phys., Diffr., Theor. Gen. Crystallogr. 24, 217 (1968).

31. E. Hovestreydt, I. Aroyo, S. Sattler, and H. Wondratschek: KAREP-A program for calculating irreducible space-group representations. J. Appl. Crystallogr. 25, 544 (1992).

32. G. Shirane: A note on the magnetic intensities of powder neutron diffraction. Acta Crystallogr. 12, 282 (1959).

33. H. Lueken: Magnetochemie. Eine Einführung in Theorie und Praxis (Teubner, Stuttgart, Germany, 1999). 